\title{
Investigation of the TP-based modeling possibility of a nonlinear ICU diabetes model
}

\author{
György Eigner*, Imre J. Rudas ${ }^{\dagger}$, and Levente Kovács* \\ ${ }^{*}$ Research and Innovation Center of Óbuda University, \\ Physiological Controls Group, Óbuda University, Budapest, Hungary \\ Email: \{eigner.gyorgy,kovacs.levente\}@nik.uni-obuda.hu \\ ${ }^{\dagger}$ Research and Innovation Center of Óbuda University, Budapest, Hungary \\ Email: rudas@uni-obuda.hu
}

\begin{abstract}
In-silico modeling is an important part of biomedical engineering. Advanced controllers providing high quality control can be validated through it checking if the available mathematical model of the given biomedical process produces the desired output. However, due to high patient variability the advanced linear control methods applied on linearized models could produce several distortions compared to the original nonlinear models; hence, these errors should be reduced. Hierarchical control strategies could be a possibility or from modeling point of view using different control-oriented modeling methodologies. Linear Parameter Varying (LPV) approaches with Linear Matrix Inequality (LMI) based modeling and controller design represent one choice. In this paper, we investigate their generalized extension, the Tensor Product (TP) model transformation demonstrated on diabetes modeling. In concrete, the type 1 diabetes modeling on Intensive Care Units (ICU) is envisaged. The achieved results will be used for TP transformation based controller design in our later work.
\end{abstract}

Index terms-Tensor Product model transformation, Modeling of diabetes, ICU model, LPV methods

\section{INTRODUCTION}

The research of potentially beneficial modeling and control methodologies in case of physiological processes has high importance. This is definitely true in case of Diabetes Mellitus (DM), where the aim is to keep the blood sugar level in a narrow range. However, the nonlinear, patient vary and time-delay processes require advanced modeling and control techniques in order to reach high quality control with good performance [1], [2]. Over the last decades the Artificial Pancreas (AP) and Intensive Care Unit (ICU) oriented modeling has been evolved [3]. In AP modeling the focus is on outpatient care, while in case of ICU modeling the goal is to improve the performance of the inpatient care reaching a tight glycaemic control. As patients are under frequent nursing care surveillance, it is enough to describe their metabolic state with roughly approximate, low-order models and not necessary to consider several possible circumstances which can be occurred in outpatient care. However, studies show that the good metabolic state can be critical from recovery point of view [4], [5].

Recently, highly developed approaches appeared regarding to modeling of diabetes. Linear Parameter Varying (LPV) techniques [6], Linear Matrix Inequality (LMI)-based methodologies [7] and the combination of these [8] correspond to the new trends of control engineering. Tensor Product (TP) model transformation can provide a unique, LPV-based way for modeling and it is well combined with LMI methods for controller design [9]. In this paper, we investigated the modeling possibility of an ICU model via TP model transformation. The paper is structured as follows: first, we introduce the TP model transformation and the used ICU model. Second, we show the possible quasi-LPV (qLPV) configurations of the applied model and the realized TP function form the models. Third, we present and discuss the results of the validations of the realized models. Finally, we overview the achievements of this study.

\section{THE TP MODEL TRANSFORMATION}

The TP model transformation based approaches originates from the parameter dependent fuzzy system techniques [10]. The TP method originally was demonstrated in [11], [12]. The approach was summarized in [9] in case of qLPV based systems and controller design. Concisely summarized, the TP transformation transforms a given function into a determined TP function form regardless from the type of the original function, if the exact transformation is possible; otherwise, the TP model transformation provides a TP function form approximation with given accuracy.

The TP form complexity can be settled by sampling frequency on the given parameter domain which allows to determine the approximation accuracy of the original function by the TP function. Since most of the qLPV models can be described by qLPV functions, TP model based transformation can be used on them. Through this process a TP transformation based TP model can be created which can approximate the original qLPV model. TP transformation is an effective way for convex hull manipulation of polytopic structures and well combined with LMI-based techniques. These properties allow 
to reach less conservative, more optimal LMI-based controller design possibilities than the usual LMI-methods [9].

A general, parameter dependent qLPV model (with $k$ states, $m$ inputs and $l$ outputs) can be given by its state space representation in the following way:

$$
\begin{aligned}
& \dot{\mathbf{x}}(t)=\mathbf{A}(\mathbf{p}(t)) \mathbf{x}(t)+\mathbf{B}(\mathbf{p}(t)) \mathbf{u}(t) \\
& \mathbf{y}(t)=\mathbf{C}(\mathbf{p}(t)) \mathbf{x}(t)+\mathbf{D}(\mathbf{p}(t)) \mathbf{u}(t) \\
& \mathbf{S}(\mathbf{p}(t))=\left(\begin{array}{ll}
\mathbf{A}(\mathbf{p}(t)) & \mathbf{B}(\mathbf{p}(t)) \\
\mathbf{C}(\mathbf{p}(t)) & \mathbf{D}(\mathbf{p}(t))
\end{array}\right)
\end{aligned}
$$

where $\mathbf{u}(t) \in \mathbb{R}^{m}$ represents the input vector, $\mathbf{y}(t) \in \mathbb{R}^{l}$ the output vector and $\mathbf{x}(t) \in \mathbb{R}^{k}$ the state vector; $\mathbf{A}(\mathbf{p}(t)) \in \mathbb{R}^{k \times k}$, $\mathbf{B}(\mathbf{p}(t)) \in \mathbb{R}^{k \times m}, \mathbf{C}(\mathbf{p}(t)) \in \mathbb{R}^{l \times k}$ and $\mathbf{D}(\mathbf{p}(t)) \in \mathbb{R}^{l \times m}$ matrices are the parameter dependent state, input, output and forward matrices, respectively.

(1a) can be written in the (1b) compact form, where $\mathbf{S}(\mathbf{p}(t)) \in \mathbb{R}^{(k+l) \times(k+m)}$ represents the parameter dependent system matrix. The parameter vector $\mathbf{p}(t) \in \Omega \in \mathbb{R}^{N}$ may vary in time. $\Omega=\left[p_{1, \min }, p_{1, \max }\right] \times\left[p_{2, \min }, p_{2, \max }\right] \times \ldots \times$ $\left[p_{N, \min }, p_{N, \text { max }}\right] \in \mathbb{R}^{N}$ forms a closed hypercube in the $N$ dimensional parameter space determined by the minimum and maximum values of the elements of the parameter vector. Hence, a parameter dependent qLPV model is well represented by its system matrix $\mathbf{S}(\mathbf{p}(t))$. In the parameter space inside the closed hypercube, the qLPV model can be described by its polytopic representation. Thus, the $\mathbf{S}(\mathbf{p}(t))$ can be described:

$$
\mathbf{S}(\mathbf{p}(t))=\sum_{r=1}^{R} w_{r}(p(t)) \mathbf{S}_{r}
$$

where $\mathbf{S}(\mathbf{p}(t))$ is given for any $\mathbf{p}(t) \in \Omega$ as the convex combinations of LTI system matrices (also known as LTI vertex systems) $\mathbf{S}_{r} \in \mathbb{R}^{(k+l) \times(k+m)}$. The finite element TP type polytopic model can be constructed via "sampling" of $\mathbf{p}(t)$ over $\Omega$ :

$$
\mathbf{S}(\mathbf{p}(t))=\sum_{i_{1}=1}^{I_{1}} \sum_{i_{2}=1}^{I_{2}} \ldots \sum_{i_{N}=1}^{I_{N}} \prod_{n=1}^{N} w_{n, i_{n}}\left(p_{n}(t)\right) \mathbf{S}_{i_{1}, i_{2}, \ldots, i_{N}}
$$

written in the following compact form [9]:

$$
\mathbf{S}(\mathbf{p}(t))=S \underset{\mathrm{n}=1}{\mathrm{~N}} \mathbf{w}_{n}\left(p_{n}(t)\right)
$$

where the $S \in \mathbb{R}^{I_{1} \times I_{2} \times \ldots \times I_{N} \times(k+l) \times(k+m)}$ coefficient tensor is created from the LTI vertex systems $\mathbf{S}_{i_{1}, i_{2}, \ldots, i_{N}}$, while the $\mathbf{w}_{n}\left(p_{n}(t)\right)$ vector consists from the $w_{n, i_{n}}\left(p_{n}(t)\right)\left(i_{n}=1 \ldots I_{N}\right)$ continuous weighting functions. The TP model will be convex if the weighting functions satisfies:

$$
\begin{aligned}
& \forall n, i, p_{n}(t): w_{n, i_{n}}\left(p_{n}(t)\right) \in[0,1] \\
& \forall n, p_{n}(t): \sum_{i=1}^{I_{n}} w_{n, i_{n}}\left(p_{n}(t)\right)=1 .
\end{aligned}
$$

More than one convex hull type can be used for TP type polytopic qLPV models. We used the Minimal Volume Simplex (MVS) type hull [13]:

$$
\mathbf{S}(\mathbf{p})=S \underset{\mathrm{n}=1}{\mathrm{~N}} \mathbf{w}^{(n)}\left(p_{n}\right)
$$

where the $S \in \mathbb{S}^{J_{1} \times \ldots \times J_{N}}$ core tensor is created from the $\mathbf{S}_{j_{1}, \ldots, j_{N}}$ matrices so that the $(S)_{j_{n}=j} n$-mode subtensors evolve a minimal volume bounding simplex for the $S \times_{n} \mathbf{w}_{j n}^{(n)}\left(p_{n}\right)$ trajectory over $n=1 . . N$.

Further details of TP transformation and applicable convex hull types can be found in [9], [13]-[15]. In order to realize TP transformation-based approaches, the TP Toolbox ${ }^{\circledR}$ for MATLAB is the most convenient solution [16].

\section{THE INVESTIGATED MODEL T1DM ICU MODEL}

ICU models can cover Type-1 DM (T1DM), Type-2 DM (T2DM) and other mixed cases [5]. In this study we investigated the T1DM-type ICU model which was developed by Wong et al [4]. The model has three states: the plasma glucose concentration $G(t)$ [mmol/L], the plasma insulin concentration $I(t)[\mathrm{mU} / \mathrm{L}]$, in which the glucose and insulin originates from external sources and the $Q(t)$ [mU/L], representing the concentration of insulin bounded to interstitial sites. Further, the model's inputs are the Carbohydrate $(\mathrm{CHO})$ intake $p(t)$ [mmol/L/min] and the external insulin $u_{e x}(t)[\mathrm{mU} / \mathrm{min}]$ input:

$$
\begin{gathered}
\dot{G}(t)=-p_{G} G(t)-S_{I}\left(G(t)+G_{E}\right) \frac{Q(t)}{1+\alpha_{G} Q(t)}+p(t) \\
\dot{Q}(t)=-k Q(t)+k I(t) \\
\dot{I}(t)=-\frac{n I(t)}{1+\alpha_{I} I(t)}+\frac{u_{e x}(t)}{V} .
\end{gathered}
$$

The descriptions of the parameters can be found in [4], [17]. The model contains unfavorable nonlinearities from mathematical point of view. Beside the product of states in (7a), two Michaelis-Menten (MM) type saturation can be found in (7a) and (7c) according to the insulin kinetics and insulin dependent glucose uptake by the given cells. The saturations are connected to the $Q$ and $I$ states.

\section{DERIVATION OF THE LPV AND TP MODELS}

\section{A. Steady state analysis}

The steady state of the model can be calculated in different ways. One of these is when the steady $G_{d}$ state and $p_{d}$ input are given. $Q_{d}, I_{d}$ and $u_{e x, d}$ can be calculated by using the (7a)-(7c) equations. An important question is the relation of $G_{d}$ to $G_{E}$. The qLPV model should approximate the system dynamics around the equilibrium points; hence, $G_{d}$ can be a "desired" equilibria and can be different from $G_{E}$. The equality of $G_{d}$ and $G_{E}$ becomes important during a TP based controller design, because the $G_{d}$ will that desired blood glucose level, what the controller has to provide. 
In the first case, we considered that $G_{d}=G_{E}$. As a result, the dynamics of the plasma glucose concentration at the equilibrium point becomes:

$$
\dot{G}(t)=0=-p_{G} G d-S_{I} 2 G_{d} \frac{Q_{d}}{1+\alpha_{G} Q_{d}}+p_{d} .
$$

With reformulation of (8), $Q_{d}$ can be calculated, as follows:

$$
\begin{gathered}
Q_{d}=\frac{-p_{G} G d+p_{d}}{S_{I} 2 G_{d}}\left(1+\alpha_{G} Q_{d}\right)=A\left(1+\alpha_{G} Q_{d}\right) \\
Q_{d}=\frac{A}{1-\alpha_{G} A} .
\end{gathered}
$$

$I_{d}$ appears by using the rearranged (10a) equation, if $Q(t)$ state is at the equilibrium point:

$$
\begin{gathered}
\dot{Q}(t)=0=-k Q_{d}+k I_{d} \\
I_{d}=Q_{d} .
\end{gathered}
$$

As a result, the dynamics of $I(t)$ at the equilibria can be described, as follows:

$$
\dot{I}(t)=0=-\frac{n I_{d}}{1+\alpha_{I} I_{d}}+\frac{u_{e x, d}}{V},
$$

from which the necessary $u_{e x, d}$ can be calculated to hold the equilibrium of the states beside the predefined $G_{d}$ and $p_{d}$ :

$$
u_{e x, d}=\frac{n I_{d}}{1+\alpha_{I} I_{d}} V .
$$

The other investigated case is when $G_{d} \neq G_{E}$. Here, only the (8), (9a) and (9b) equations will be different. Naturally, the numerical values of $I_{d}, Q_{d}$ and $u_{e x, d}$ will change accordingly:

$$
\dot{G}(t)=0=-p_{G} G d-S_{I}\left(G_{d}+G_{E}\right) \frac{Q_{d}}{1+\alpha_{G} Q_{d}}+p_{d}
$$

By rearranging (8), $Q_{d}$ can be calculated as follows:

$$
\begin{gathered}
Q_{d}=\frac{-p_{G} G d+p_{d}}{S_{I}\left(G_{d}+G_{E}\right)}\left(1+\alpha_{G} Q_{d}\right)=B\left(1+\alpha_{G} Q_{d}\right) \\
Q_{d}=\frac{B}{1-\alpha_{G} B} .
\end{gathered}
$$

\section{B. Investigated $q L P V$ models}

In this study we investigated different approaches as more than one realizable qLPV form can be derived.

We consider the two above-mentioned cases: $G_{d}=G_{E}$ and $G_{d} \neq G_{E}$. Many options can be selected as aim of TP-based control. One of them is when the aim of the controller is to prevent the system's diversion from the selected equilibrium point; or if the diversion becomes to provide fast action leading the system back to the equilibrium. A natural way to describe this evasive error dynamics is if we take the difference of the actual states and the steady states.
First, we consider the $G_{d}=G_{E}$ case. The error dynamics becomes as follows (subtracting $S_{I} G(t) \frac{Q_{d}}{1+\alpha_{G} Q_{d}}$ from the last two parts of (15):

$$
\begin{aligned}
& \Delta \dot{G}(t)=\dot{G}(t)-0= \\
& -p_{G} G(t)-S_{I}\left(G(t)+G_{d}\right) \frac{Q(t)}{1+\alpha_{G} Q(t)}+p(t) \\
& -\left[-p_{G} G_{d}-S_{I} 2 G_{d} \frac{Q_{d}}{1+\alpha_{G} Q_{d}}+p_{d}\right]= \\
& -p_{G} G(t)+p_{G} G_{d}+p(t)-p_{d}- \\
& S_{I} G(t) \frac{Q(t)}{1+\alpha_{G} Q(t)}-S_{I} G_{d} \frac{Q(t)}{1+\alpha_{G} Q(t)}+ \\
& S_{I} G_{d} \frac{Q_{d}}{1+\alpha_{G} Q_{d}}+S_{I} G_{d} \frac{Q_{d}}{1+\alpha_{G} Q_{d}}= \\
& -p_{G}\left(G(t)-G_{d}\right)+\left(p(t)-p_{d}\right)- \\
& S_{I} G_{d}\left(\frac{Q(t)}{1+\alpha_{G} Q(t)}-\frac{Q_{d}}{\left.1+\alpha_{G} Q_{d}\right)}\right)- \\
& S_{I} G(t) \frac{Q(t)}{1+\alpha_{G} Q(t)}+S_{I} G_{d} \frac{Q_{d}}{1+\alpha_{G} Q_{d}}= \\
& -p_{G} \Delta G(t)+\Delta p(t)- \\
& S_{I} G_{d} \frac{1}{\left(1+\alpha_{G} Q(t)\right)\left(1+\alpha_{G} Q_{d}\right)} \Delta Q(t)- \\
& S_{I} G(t) \frac{Q(t)}{1+\alpha_{G} Q(t)}+S_{I} G_{d} \frac{Q_{d}}{1+\alpha_{G} Q_{d}} \\
& -S_{I} G(t) \frac{Q(t)}{1+\alpha_{G} Q(t)}+S_{I} G_{d} \frac{Q_{d}}{1+\alpha_{G} Q_{d}}+ \\
& S_{I} G(t) \frac{Q_{d}}{1+\alpha_{G} Q_{d}}-S_{I} G(t) \frac{Q_{d}}{1+\alpha_{G} Q_{d}}= \\
& -S_{I} G(t)\left(\frac{Q(t)}{1+\alpha_{G} Q(t)}-\frac{Q_{d}}{\left.1+\alpha_{G} Q_{d}\right)}\right)- \\
& -S_{I} \frac{Q_{d}}{1+\alpha_{G} Q_{d}}\left(G(t)-G_{d}\right)= \\
& -S_{I} G(t) \frac{1}{\left(1+\alpha_{G} Q(t)\right)\left(1+\alpha_{G} Q_{d}\right)} \Delta Q(t)- \\
& -S_{I} \frac{Q_{d}}{1+\alpha_{G} Q_{d}} \Delta G(t)
\end{aligned}
$$

From here, the error dynamics of $G$ state at the equilibrium point can be described as:

$$
\begin{aligned}
& \Delta \dot{G}(t)=-\left(p_{G}+S_{I} \frac{Q_{d}}{1+\alpha_{G} Q_{d}}\right) \Delta G(t)- \\
& S_{I}\left(G(t)+G_{d}\right) \frac{1}{\left(1+\alpha_{G} Q(t)\right)\left(1+\alpha_{G} Q_{d}\right)} \Delta Q(t)+\Delta p(t)
\end{aligned}
$$

The second case is when $G_{d} \neq G_{E}$. In this case, the error dynamics $\Delta G(t)$ becomes as follows:

$$
\begin{aligned}
& \Delta \dot{G}(t)=-\left(p_{G}+S_{I} \frac{Q_{d}}{1+\alpha_{G} Q_{d}}\right) \Delta G(t)- \\
& S_{I}\left(G(t)+G_{E}\right) \frac{1}{\left(1+\alpha_{G} Q(t)\right)\left(1+\alpha_{G} Q_{d}\right)} \Delta Q(t)+\Delta p(t)
\end{aligned}
$$


As a result, the error dynamics of the $Q(t)$ and $I(t)$ can be easily derived as in (17):

$$
\begin{aligned}
& \Delta \dot{Q}(t)=\dot{Q}(t)-0 \\
& -k Q(t)+k I(t)-\left[-k Q_{d}+k I_{d}\right]= \\
& -k\left(Q(t)-Q_{d}\right)+k\left(I(t)-I_{d}\right)= \\
& \Delta \dot{Q}(t)=-k \Delta Q(t)+k \Delta I(t)
\end{aligned}
$$

$$
\begin{aligned}
& \Delta \dot{I}(t)=\dot{I}(t)-0= \\
& -n \frac{I(t)}{1+\alpha_{I} I(t)}+\frac{u_{e x}}{V}-\left[-n \frac{I_{d}}{1+\alpha_{I} I_{d}}+\frac{u_{e x, d}}{V}\right]= \\
& -n\left(\frac{I(t)}{1+\alpha_{I} I(t)}-\frac{I_{d}}{\left.1+\alpha_{I} I_{d}\right)}\right)+\frac{1}{V}\left(u_{e x}-u_{e x, d}\right)= \\
& \Delta \dot{I}(t)=-n \frac{1}{\left(1+\alpha_{I} I(t)\right)\left(1+\alpha_{I} I_{d}\right)} \Delta I(t)+\frac{1}{V} \Delta u_{e x}(t)
\end{aligned}
$$

A convenient way is the idea if the error dynamics-based qLPV models are represented with their state-space form. In this way the inputs can be separated: the control input becomes the external insulin intake $u_{e x}(t)$, while the disturbance is the $p(t)$ external $\mathrm{CHO}$ intake. We switched the order of the inputs in sake of clarity, namely, the first input in the state-space representation is the insulin intake $u_{e x}(t)$, while the second is the $\mathrm{CHO}$ disturbance $p(t)$. As the goal is to describe the error dynamics, the difference between the actual input and steady inputs should be considered. In this way, the inputs are: $\Delta u(t)=\left[\Delta u_{e x}(t), \Delta p(t)\right]^{T}$. The states of the qLPV models are based on the error dynamics, namely $\Delta x(t)=[\Delta G(t), \Delta Q(t), \Delta I(t)]^{T}$. From these considerations and the (17)-(20) equations, the state-space representations of the derived qLPV models are represented by (22) considering $G_{d}=G_{E}$ and (23) considering $G_{d} \neq G_{E}$.

\section{TP models}

The TP model transformation can be applied on the qLPV system matrices $\mathbf{S}\left\{\left.(G(t), Q(t), I(t))\right|_{G_{d}=G_{E}}\right\}$ of (22) and $\mathbf{S}\left\{\left.(G(t), Q(t), I(t))\right|_{G_{d} \neq G_{E}}\right\}$ of (23). The transformation provides the following TP model structure:

$$
\begin{aligned}
& \mathbf{S}\left\{\left.(G(t), Q(t), I(t))\right|_{G_{d}=G_{E}}\right\}=S \underset{\mathrm{n}=1}{\stackrel{3}{\otimes}} \mathbf{w}_{n}\left(p_{n}(t)\right)= \\
& S \times_{1} \mathbf{w}_{1}(G(t)) \times_{2} \mathbf{w}_{2}(Q(t)) \times_{3} \mathbf{w}_{3}(I(t)) \\
& \mathbf{S}\left\{\left.(G(t), Q(t), I(t))\right|_{G_{d} \neq G_{E}}\right\}=S \underset{\mathrm{n}=1}{\stackrel{3}{\otimes}} \mathbf{w}_{n}\left(p_{n}(t)\right)= \\
& S \times_{1} \mathbf{w}_{1}(G(t)) \times_{2} \mathbf{w}_{2}(Q(t)) \times_{3} \mathbf{w}_{3}(I(t))
\end{aligned}
$$

Figure 1 shows the MVS-type weighting functions with dense sampling (left column belongs to (21a) and the right column belongs to (21b)). There are no evaluable difference between the given weighting functions; however, small numerical differences appeared.
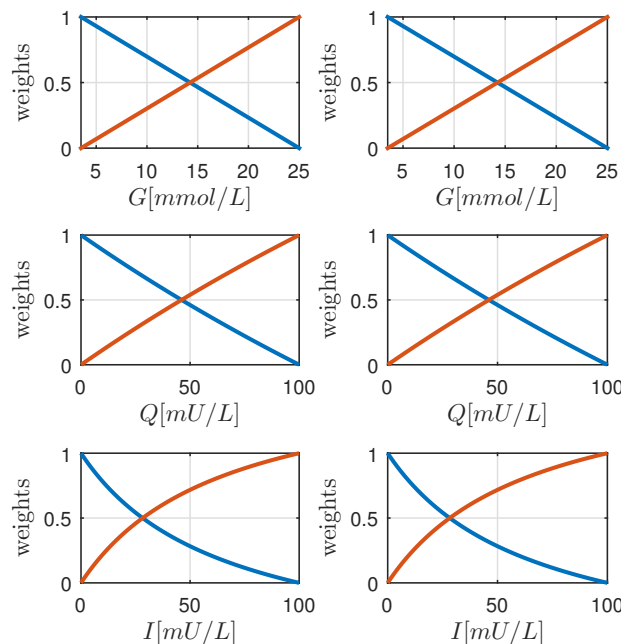

Figure 1. Weighting functions of the TP polytopic model. Left column: $\mathbf{w}_{n}(p(t))_{G_{d}=G_{E}}$, right column: $\mathbf{w}_{n}(p(t))_{G_{d} \neq G_{E}}$

\section{VALIDATION AND DISCUSSION}

In order to validate the generated models, we built up a validation environment in MATLAB which is able to make the comparisons between the original and realized qLPV and TP models automatically.

$$
\begin{aligned}
& \Delta \dot{x}(t)=\left[\begin{array}{ccc}
-\left(p_{G}+\frac{S_{I} Q_{d}}{1+\alpha_{G} Q_{d}}\right) & \frac{-S_{I}\left(G(t)+G_{d}\right)}{\left(1+\alpha_{G} Q(t)\right)\left(1+\alpha_{G} Q_{d}\right)} & 0 \\
0 & -k & k \\
0 & 0 & \frac{-n}{\left(1+\alpha_{I} I(t)\right)\left(1+\alpha_{I} I_{d}\right)}
\end{array}\right] \Delta x(t)+\left[\begin{array}{cc}
0 & 1 \\
0 & 0 \\
\frac{1}{V} & 0
\end{array}\right] \Delta u(t) \\
& \Delta \dot{x}(t)=\left[\begin{array}{ccc}
-\left(p_{G}+\frac{S_{I} Q_{d}}{1+\alpha_{G} Q_{d}}\right) & \frac{-S_{I}\left(G(t)+G_{E}\right)}{\left(1+\alpha_{G} Q(t)\right)\left(1+\alpha_{G} Q_{d}\right)} & 0 \\
0 & -k & k \\
0 & 0 & \frac{-n}{\left(1+\alpha_{I} I(t)\right)\left(1+\alpha_{I} I_{d}\right)}
\end{array}\right] \Delta x(t)+\left[\begin{array}{cc}
0 & 1 \\
0 & 0 \\
\frac{1}{V} & 0
\end{array}\right] \Delta u(t)
\end{aligned}
$$


The main considerations during the validation were the following:

1) Investigated parameter domain: $G=3.5 . .25, Q=0 . .100$ and $I=0 . .100$;

2) Comparison was done between every state of every model;

3) Dense (considered number of samples $(\mathrm{NoS}): N o S_{G}=$ $\left.31, N o S_{Q}=101, N o S_{I}=101\right)$ and less dense $\left(N o S_{G}=\right.$ $\left.17, N o S_{Q}=81, N o S_{I}=81\right)$ parameter sampling in the parameter domain;

4) Comparison only in case of initial state decay and in case of given inputs;

5) Use of Root-Mean Square Error (RMSE) as basis of comparison.

The results of the validation are summarized in Table I. In every subtable the upper triangular partition belongs to the dense sampling, namely, the number of samples (NoS) were higher on the investigated parameter domain. The model notation is the following:

- original nonlinear model: Original (7);

- qLPV model of (22): $q L P V_{1}$;

- qLPV model of (23): $q L P V_{2}$;

- TP model of (21a): $T P_{1}$;

- TP model of (21b): $T P_{2}$.

In case of Table Ia, a less than 100 minutes decay was investigated for the initial values of the state variables. The difference between dense and less dense sampling is negligible. However, both TP models had small RMSE at the given circumstances, but the $T P_{1}$ model where $G_{d}=G_{E}$ had the best performance.

Table Ib shows a scenario, where external $\mathrm{CHO}$ and insulin inputs were impulse functions (similar to reality), as follows:

- CHO intake: Height: 4 g, Width: 5 min, Period: 50 min

- Insulin intake: Height: $1 \mathrm{U}$, Width: $2 \mathrm{~min}$, Period: $50 \mathrm{~min}$

We transformed the inputs from $g$ to $\mathrm{mmol} / \mathrm{L}(\mathrm{CHO})$ and $U$ to $m U / L$ (insulin) based on the model parameters in Table I. The density of sampling did not cause evaluable difference in the resulting RMSE of the states based on the data. In this case, $T P_{2}$ model produced the smallest RMSE under 300 minutes.

Figure 2 shows the results of the second investigation (as in Table Ib) in case of dense sampling. It can be considered, that the variation of $Q(t)$ and $I(t)$ are almost the same. However, the $T P_{2}$ model proved to be much more accurate than the $T P_{1}$ in the $G(t)$ state, as the $G_{O r i g}(t)$ and $G_{T P_{2}}(t)$ states overlap each other.

On Figure 2 the error of the states were highlighted in such a way, that the state variation of the realized TP models were subtracted from the original states. The results confirmed the numerical RMSE-based evaluation in Table Ib and one can see that $T P_{1}$ is more suitable to substitute the original nonlinear model.
Table I

RESULTS OF THE RMSE-BASED INVESTIGATIONS. USED PARAMETER SET: $G_{E}=10.5 \mathrm{MMOL} / \mathrm{L}, p_{G}=0.011 / \mathrm{MIN}, S_{I}=0.001 \mathrm{~L} / \mathrm{MU} / \mathrm{MIN}, V=12$ $\mathrm{L}, k=0.01981 / \mathrm{MIN}, n=0.161 / \mathrm{MIN}, \alpha_{I}=0.0017 \mathrm{~L} / \mathrm{MU}$ AND $\alpha_{G}=0.0154$ $\mathrm{L} / \mathrm{MU}$.

Investigation 1: RMSE-based comparison of the states of the realized models on the given parameter domain under 100 minutes. Initial conditions: $G_{0}=15$, $Q_{0}=3$ and $I_{0}=5$

\begin{tabular}{|c|c|c|c|c|c|c|}
\hline & \multicolumn{5}{|c|}{$\mathbf{G}[\mathrm{mmol} / \mathrm{L}]$} \\
\hline & & \multicolumn{5}{|c|}{$\mathrm{NoS}=31$} \\
\hline & & Original & $q L P V_{1}$ & $q L P V_{2}$ & $T P_{1}$ & $T P_{2}$ \\
\hline \multirow{5}{*}{$\begin{array}{l}\text { II } \\
\text { है }\end{array}$} & Original & & 1.4295 & 0.0982 & 0.0469 & 0.1273 \\
\hline & $q L P V_{1}$ & 1.4295 & & 1.3278 & 1.3826 & 1.5568 \\
\hline & $q L P V_{2}$ & 0.0982 & 1.5278 & & 0.1452 & 0.0290 \\
\hline & $T P_{1}$ & 0.0469 & 1.3826 & 0.1452 & & 0.1743 \\
\hline & $T P_{2}$ & 0.1273 & 1.5569 & 0.0291 & 0.1742 & \\
\hline
\end{tabular}

\begin{tabular}{|c|c|c|c|c|c|c|}
\hline & \multicolumn{5}{|c|}{$\mathrm{Q}[\mathrm{mU} / \mathrm{L}]$} \\
\hline & & \multicolumn{5}{|c|}{$\mathrm{NoS}=101$} \\
\hline & & Original & $q L P V_{1}$ & $q L P V_{2}$ & $\overline{T P_{1}}$ & $T P_{2}$ \\
\hline \multirow{5}{*}{ 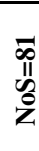 } & Original & & 0.0051 & 0.0051 & 0.0022 & 0.0051 \\
\hline & $q L P V_{1}$ & 0.0051 & & 0 & 0.0073 & 0 \\
\hline & $q L P V_{2}$ & 0.0051 & 0 & & 0.0073 & 0 \\
\hline & $T P_{1}$ & 0.0022 & 0.0073 & 0.0073 & & 0.0073 \\
\hline & $T P_{2}$ & 0.0051 & 0 & 0 & 0.0073 & \\
\hline
\end{tabular}

\begin{tabular}{|c|c|c|c|c|c|c|}
\hline & \multicolumn{4}{|c|}{ I [mU/L] } & \\
\hline & & \multicolumn{5}{|c|}{$\mathrm{NoS}=101$} \\
\hline & & Original & $q L P V_{1}$ & $q L P V_{2}$ & $\overline{T P_{1}}$ & $T P_{2}$ \\
\hline \multirow{5}{*}{$\begin{array}{l}\text { क् } \\
\text { II } \\
\text { ठ } \\
z\end{array}$} & Original & & 0.0031 & 0.0031 & 0.0052 & 0.0030 \\
\hline & $q L P V_{1}$ & 0.0031 & & $\overline{0}$ & 0.0083 & $\overline{0}$ \\
\hline & $q L P V_{2}$ & 0.0031 & 0 & & 0.0083 & 0 \\
\hline & $\overline{T P_{1}}$ & 0.0052 & 0.0083 & 0.0083 & & 0.0083 \\
\hline & $T P_{2}$ & 0.0030 & 0 & 0 & 0.0083 & \\
\hline
\end{tabular}

Table Ia.

Investigation 2: RMSE-based comparison of the states of the realized models on the given parameter domain under 300 minutes beside given impulse-kind inputs. Initial conditions: $G_{0}=15, Q_{0}=3$ and $I_{0}=5$

\begin{tabular}{|c|c|c|c|c|c|c|}
\hline & \multicolumn{6}{|c|}{$\mathrm{G}[\mathrm{mmol} / \mathrm{L}]$} \\
\hline & & \multicolumn{5}{|c|}{ NoS $=31$} \\
\hline & & Original & $q L P V_{1}$ & $q L P V_{2}$ & $T P_{1}$ & $T P_{2}$ \\
\hline \multirow{5}{*}{$\begin{array}{l}\text { त } \\
\text { है } \\
z\end{array}$} & Original & & 2.3666 & 0.0339 & 1.3614 & 0.0244 \\
\hline & $q L P V_{1}$ & 2.3666 & & 2.4005 & 1.0052 & 2.391 \\
\hline & $q L P V_{2}$ & 0.0339 & 2.4005 & & 1.3953 & 0.0095 \\
\hline & $T P_{1}$ & 1.3611 & 1.0055 & 1.3950 & & 1.3858 \\
\hline & $T P_{2}$ & 0.0246 & 1.0055 & 0.0093 & 1.3857 & \\
\hline
\end{tabular}

\begin{tabular}{|c|c|c|c|c|c|c|}
\hline & \multicolumn{5}{|c|}{$\mathrm{Q}[\mathrm{mU} / \mathrm{L}]$} \\
\hline & & \multicolumn{5}{|c|}{ NoS $=101$} \\
\hline & & Original & $q L P V_{1}$ & $q L P V_{2}$ & $T P_{1}$ & $T P_{2}$ \\
\hline \multirow{5}{*}{ 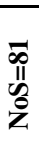 } & Original & & 0.0127 & 0.0127 & 0.0254 & 0.0125 \\
\hline & $q L P V_{1}$ & 0.0127 & & 0 & 0.0127 & 0.0002 \\
\hline & $q L P V_{2}$ & 0.0127 & $\overline{0}$ & & 0.0127 & 0.0002 \\
\hline & $T P_{1}$ & 0.0254 & 0.0127 & 0.0127 & & 0.0129 \\
\hline & $T P_{2}$ & 0.0125 & 0.0002 & 0.0002 & 0.0129 & \\
\hline
\end{tabular}

\begin{tabular}{|c|c|c|c|c|c|c|}
\hline & \multicolumn{5}{|c|}{ I [mU/L] } \\
\hline & & \multicolumn{5}{|c|}{$\mathrm{NoS}=101$} \\
\hline & & Original & $q L P V_{1}$ & $q L P V_{2}$ & $T P_{1}$ & $T P_{2}$ \\
\hline \multirow{5}{*}{$\begin{array}{l}\bar{\infty} \\
\text { II } \\
0 \\
\text { Z }\end{array}$} & Original & & 0.0088 & 0.0088 & 0.0005 & 0.0089 \\
\hline & $q L P V_{1}$ & 0.0088 & & 0 & 0.0083 & 0.0001 \\
\hline & $q L P V_{2}$ & 0.0088 & 0 & & 0.0083 & 0.0001 \\
\hline & $T P_{1}$ & 0.0005 & 0.0083 & 0.0083 & & 0.0084 \\
\hline & $T P_{2}$ & 0.0089 & 0.0001 & 0.001 & 0.0084 & \\
\hline
\end{tabular}

Table Ib. 

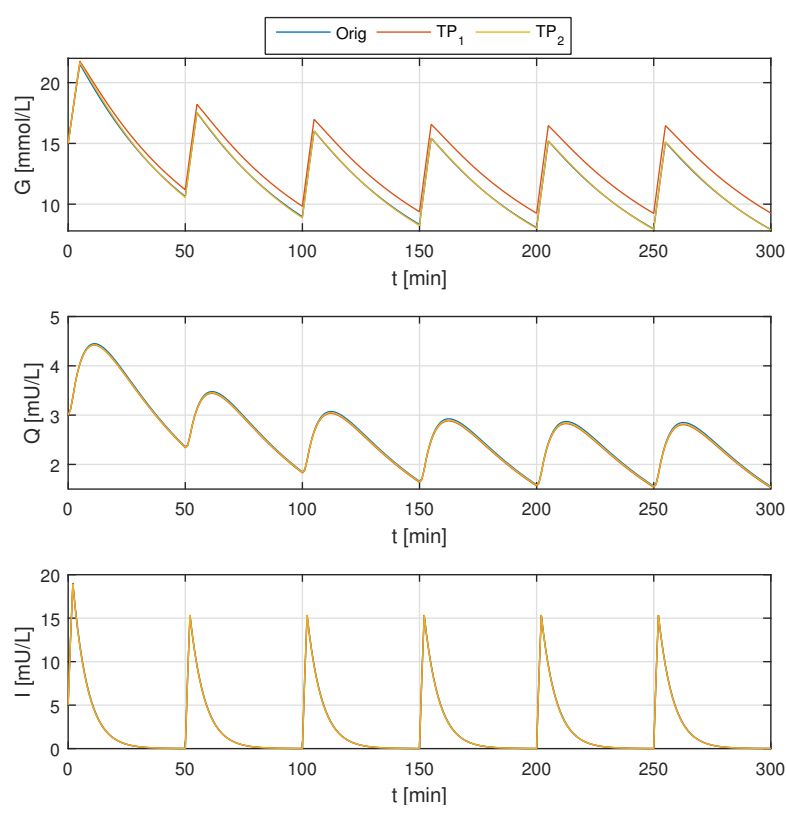

Figure 2. 300 minutes long simulation in case of realistic inputs.
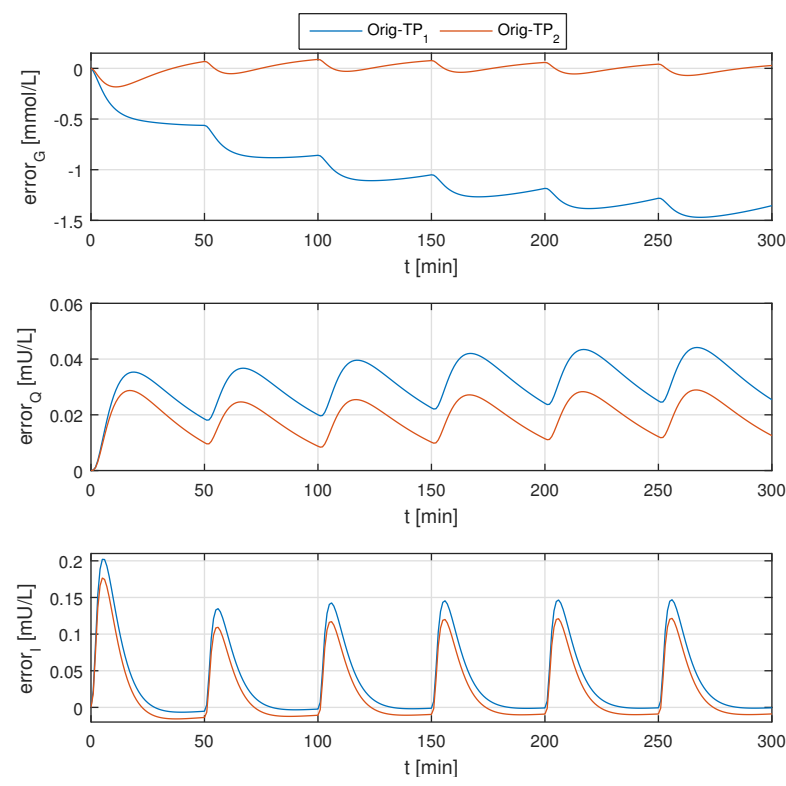

Figure 3. State error evolution over 300 minutes long simulation in case of realistic inputs.

\section{CONCLUSION}

In this paper we investigated the applicability of TP model transformation in case of a well-known ICU diabetes model in order to realize different TP models. We examined two cases: the TP model, when the "operating equilibrium of glycemia $\left(G_{d}\right)$ " of the model was considered equal to the model equilibrium of glycemia $\left(G_{E}\right)$ and were it was not. We found based on numerical validation that in case of realistic simulations we can reach better performance, namely, smaller difference between the realized TP model and the original model, when the operating equilibrium is not equal to the model equilibrium. Further work will focus control design of the realized TP model via the LMI-based TP controller design method. This advanced tool let to embed several criteria and constraints to the control structure and results reliable and robust controller for the given TP model. TP model can be robust itself, if more parameters are included into the parameter vector. We will investigate this possibility also.

\section{ACKNOWLEDGMENT}

The authors thankfully acknowledge the support of the Robotics Special College of Obuda University. The research was also supported by the Research and Innovation Center of Obuda University and the European Research Council Starting Grant ERC-StG 679681.

\section{REFERENCES}

[1] J. Bronzino and D. Peterson, The Biomedical Engineering Handbook, 4th ed. Boca Raton, Florida, USA: CRC Press, 2015.

[2] M. Messori, C. Cobelli, and L. Magni, "Artificial pancreas: from insilico to in-vivo," in 9th International Symposium on Advanced Control of Chemical Processes. IFAC, 2014, pp. $1301-1309$.

[3] V.N. Shah, A. Shoskes, B. Tawfik, and S.K. Garg, "Closed-loop system in the management of diabetes: Past, present, and future," Diabetes Technol The, vol. 16, no. 8, pp. 477-490, 2014.

[4] X. Wong, J. Chase, G. Shaw, C. Hanna, T. Lotz, J. Lin, I. Singh-Levett, L. Hollingsworth, and O. Wong, "Model predictive glycaemic regulation in critical illness using insulin and nutrition input: A pilot study," Med Eng Phys, vol. 28, pp. 665 - 681, 2006.

[5] J.G. Chase, A.J. Le Compte, J.G. Preiser, G.M.. Shaw, S. Penning, and T. Desaive, "Physiological modeling, tight glycemic control, and the icu clinician: what are models and how can they affect practice?" Ann Intensive Care, vol. 1, no. 11, pp. P1-8, 2011.

[6] Gy. Eigner, J.K. Tar, I. Rudas, and L. Kovacs, "LPV-based quality interpretations on modeling and control of diabetes," ACTA Pol Hung, vol. 13, no. 1, pp. 171 - 190, 2016.

[7] P. Palumbo, S. Ditlevsen, A. Bertuzzi, and A. de Gaetano, "Mathematical modeling of the glucose-insulin system a review," Math Biosci, vol. 244, no. 2, pp. 69-81, 2013.

[8] P. Szalay, Gy. Eigner, and L. Kovacs, "Linear matrix inequality-based robust controller design for type-1 diabetes model," in IFAC 2014 - 19th World Congress of The International Federation of Automatic Control. IFAC, 2014, pp. 9247 - 9252.

[9] P. Baranyi, Y. Yam, and P. Varlaki, Tensor Product Model Transformation in Polytopic Model-Based Control, 1st ed., ser. Series: Automation and Control Engineering. Boca Raton, USA: CRC Press, 2013.

[10] K. Tanaka and H. O. Wang, Fuzzy Control Systems Design and Analysis: A Linear Matrix Inequality Approach, 1st ed. Chichester, UK: John Wiley and Sons, 2001

[11] P. Baranyi, D. Tikk, Y. Yam, and R. Patton, "TP model transformation as a way to lmi-based controller design," Comput Ind, vol. 51, no. 3, pp. $281-297,2003$.

[12] P. Baranyi, "TP model transformation as a way to lmi-based controller design," IEEE T Ind Electron, vol. 51, no. 2, pp. 387 - 400, 2004.

[13] J. Kuti, P. Galambos, and P. Baranyi, "Minimal volume simplex (mvs) approach for convex hull generation in tp model transformation," in 2014 18th International Conference on Intelligent Engineering Systems (INES 2014). IEEE Hungary Section, pp. 187 - 192.

[14] — "Minimal volume simplex (mvs) convex hull generation and manipulation methodology for tp model transformation," Asian J Control, 2015, submitted.

[15] P. Galambos and P. Baranyi, "TP $\tau$ model transformation: A systematic modelling framework to handle internal time delays in control systems," Asian J Control, vol. 17, no. 2, pp. 1 - 11, 2015.

[16] MTA SZTAKI. (2016) TPtool - Tensor Product MATLAB Toolbox. [Online]. Available: http://tptool.sztaki.hu

[17] X. Wong, J. Chase, G. Shaw, C. Hann, J. Lin, and T. Lotz, "Comparison of adaptive and sliding-scale glycaemic control in critical care and the impact of nutritional inputs," in 12th International Conference On Biomedical Engineering, 2005, pp. $1-4$. 